$\underline{\text { Research Articles }}$

\title{
Prevalence and patterns of chronic communicable and non- communicable diseases multimorbidity in sub-Saharan Africa: protocol for a systematic review
}

\author{
Mpho R Disang ${ }^{1}$, David Weller ${ }^{2}$, Christine Campbell ${ }^{2}$ \\ 1 Usher Institute, University of Edinburgh, Edinburgh, UK; Department of Population Studies, University of Botswana, Gaborone, Botswana, ${ }^{2}$ Usher \\ Institute, University of Edinburgh, Edinburgh, UK \\ Keywords: sub-saharan africa, communicable diseases, non communicable diseases, multimorbidity \\ https://doi.org/10.29392/001c.21340
}

Journal of Global Health Reports

Vol. 5, 2021

\begin{abstract}
Background
The burden of multimorbidity is rising globally. In sub-Saharan Africa (SSA), the growing prevalence of non-communicable diseases alongside the already existing high burden of chronic communicable diseases such as human immunodeficiency virus (HIV) presents a complex form of multimorbidity relative to patterns observed in high income countries. There is a gap in understanding the true burden of multimorbidity in SSA, in particular at interface of chronic communicable and non-communicable diseases (NCDs). The primary objective of this systematic review is to identify, evaluate and summarise the evidence on the prevalence and patterns of chronic communicable and NCDs multimorbidity in SSA.
\end{abstract}

\begin{abstract}
Methods and analysis
This protocol was developed in compliance with the Preferred Reporting Items for Systematic Review and Meta-Analyses Protocols (PRISMA-P) guidelines. A comprehensive search will be conducted on selected electronic databases including Medline, Embase, African Journals Online, based on a predefined search strategy for observational studies reporting primary data on multimorbidity from any country in the SSA region. Additionally, we will explore grey literature databases and check reference lists of included studies. Study selection, assessment of quality of included studies and data extraction will be carried out independently by two reviewers. A narrative synthesis will be conducted and meta-analysis will be carried out where applicable.
\end{abstract}

\section{Conclusion}

The proposed systematic review will provide insights into the interface of chronic communicable diseases and NCDs in SSA. We expect this will provide additional insights into the true picture of multimorbidity in the region and assist policy makers to design appropriate prevention and management strategies. This review does not require ethical approval. Upon completion, it will be submitted for publication in peer-reviewed journals, and disseminated through relevant academic conferences.

Prospero registration: CRD42020183539

Global measures to reduce premature mortality have contributed to a rise in life expectancy: people live longer and a growing proportion live with more than one chronic condition, a phenomenon commonly referred to as multimorbidity. ${ }^{1}$ Previous research has found that individuals with multiple chronic conditions have complex healthcare needs and are characterised by deteriorated physical, social and mental wellbeing, higher premature mortality, poor quality of life and increased utilisation of healthcare and social services. ${ }^{2,3}$ One of the largest prevalence studies established that multimorbidity is associated with low socioeconomic status and that individuals from deprived areas were likely to develop multiple chronic conditions ten to fifteen years earlier than those from more affluent settings. ${ }^{4}$
Although most evidence comes from high income countries (HICs), ${ }^{5}$ it is evident that the burden of multimorbidity is rising and putting pressure on health care systems and economies worldwide. ${ }^{6}$ Prevalence estimates range from below $15 \%$ to above $95 \% .{ }^{7}$ A recent systematic review summarising global literature on the prevalence of multimorbidity based on seventy community based studies, estimated a prevalence of $37.9 \%$ for HICs and $29.7 \%$ in lowand middle-income countries (LMICs). ${ }^{8}$ Understanding and mapping the magnitude of multimorbidity, as well as how diseases co-exist is critical in allocation of resources in healthcare and development of effective, efficient evidencebased management and prevention strategies. ${ }^{8,9}$ The literature has pointed to the lack of international consensus on 
the operational definition of multimorbidity as well as the selection of conditions that meet the chronicity criteria as the major reason behind varying results that are difficult to compare across different settings. 1,7,10

\section{MULTIMORBIDITY IN THE SUB-SAHARAN AFRICA CONTEXT}

Although many countries especially HICs have managed to significantly reduce the prevalence of communicable diseases, in the sub-Saharan Africa region there remains a significant burden from diseases such as tuberculosis (TB), human immunodeficiency virus (HIV), malaria and these remain the leading causes of mortality and morbidity. ${ }^{11,12}$ In parallel, the prevalence of NCDs in SSA is rising - primarily cardiovascular diseases, chronic respiratory disease, diabetes, mental disorders and cancers. Due to rapid urbanisation, adoption of risky lifestyles such as harmful use of tobacco and alcohol, lack of exercise and poor diet, the region has experienced a rise of approximately $67 \%$ in the burden of NCDs between 1990 and 2017. ${ }^{13}$ Furthermore, due to the success of antiretroviral treatment (ART), survival in people living with HIV has significantly improved ${ }^{14}$ and research shows that these individuals have an increased risk of developing chronic conditions such as cardiovascular diseases, depression, cancers among others. ${ }^{15,16}$

This increased prevalence of NCDs among people living with HIV has been linked to various factors including the nature of HIV infection, effects of certain antiretroviral drugs, ageing and other well-known NCD risk factors. ${ }^{17}$ This interaction presents a complex form of multimorbidity that is different from the one observed in HICs. For example, SSA is likely to have a larger proportion of an ageing population on ART in the coming decades. ${ }^{18}$ This highlights the importance of generating context-specific evidence for SSA, a region with the highest global burden of HIV infection which is, concurrently, experiencing a rapid increase in the burden of NCDs.

\section{RATIONALE}

Although there is clear evidence that the prevalence of multiple chronic conditions is rising in all regions of the world including sub-Saharan Africa (SSA), there is still limited evidence from the region to give a clear picture of the status or burden of multimorbidity. Most systematic reviews on prevalence and patterns of multimorbidity to date have focused on HICs and those that included SSA countries grouped them together with other countries in the 'LMIC' category. Evidence is needed specifically for this region due to the unique disease profile of a double burden of communicable diseases and NCDs. Given the rapid population growth in the region, an ageing HIV-positive population, the interaction of NCDs and communicable diseases, and already existing health care challenges, it is imperative to generate SSA-specific evidence.

The overall aim of this review is to identify and synthesise the evidence on the prevalence and patterns of chronic disease multimorbidity among adults in sub Saharan Africa by examining the interaction of chronic communicable diseases such as HIV, TB with the most prevalent NCDs in SSA.
We will also evaluate the evidence on how multimorbidity in the region is typically measured, and how the differences in measurement might influence reported patterns of multimorbidity. With the advent of the COVID-19 pandemic, we will also incorporate emerging literature on the impact of this pandemic on chronic conditions. To the best of our knowledge, this is the first systematic review that will map evidence on the combined burden of non-communicable and communicable disease multimorbidity specifically for SSA region.

\section{METHODS}

The development and reporting of this protocol was in accordance with the Preferred Reporting Items for Systematic Review and Meta-Analyses Protocols (PRISMA-P) 2015 statement. ${ }^{19}$ This protocol has been published with the International Prospective Register for Systematic Reviews (PROSPERO), registration number CRD42020183539.

\section{ELIGIBILITY CRITERIA}

The inclusion criteria is guided by the CoCoPop (condition, context and population) framework for reviews of prevalence studies recommended by the Joanna Briggs Institute. ${ }^{20,21}$

Condition: We will adopt the Academy of Medical Sciences definition of multimorbidity: the coexistence of two or more chronic conditions, each one of which is either; a physical non-communicable disease of long duration, such as a cardiovascular disease or cancer, a mental health condition of long duration, such as a mood disorder or dementia, or an infectious disease of long duration, such as HIV or hepatitis $\mathrm{C} .{ }^{22}$

Context: We will consider studies from the sub-Saharan Africa region. Sub-Saharan Africa will be defined according to the World Bank country classification ${ }^{23}$ as illustrated in Table 1.

Population: We will include multimorbidity studies involving adults aged 18 years and older.

Studies will be selected based on the following inclusion criteria;

1. Original studies aimed at estimating the prevalence of multimorbidity or documenting the frequent disease patterns conducted either at community or health facility level.

2. Observational studies including cross sectional and longitudinal studies. For longitudinal studies we will extract prevalence at baseline.

3. Government and non-governmental surveillance data/reports and theses that report original data on prevalence or patterns of multimorbidity.

Due to the paucity of research on multimorbidity in subSaharan Africa, studies that selected participants based on a specific condition (comorbidity studies) will not be excluded since it is important to understand how the two concepts have been defined and measured in the region. Distinctions on the characteristics of comorbidity and multimorbidity studies will be reported accordingly.

We will collect data from studies published between Jan- 
Table 1. Sub-Saharan Africa (World Bank country classification)

\begin{tabular}{|l|l|l|}
\hline Angola & Ethiopia & Niger \\
\hline Benin & Gabon & Nigeria \\
\hline Botswana & Gambia, The & Rwanda \\
\hline Burkina Faso & Ghana & São Tomé and Principe \\
\hline Burundi & Guinea & Senegal \\
\hline Cabo Verde & Guinea-Bissau & Seychelles \\
\hline Cameroon & Kenya & Sierra Leone \\
\hline Central African Republic & Lesotho & Somalia \\
\hline Chad & Liberia & South Africa \\
\hline Comoros & Madagascar & South Sudan \\
\hline Congo, Dem. Rep. & Malawi & Sudan \\
\hline Congo, Rep & Mali & Tanzania \\
\hline Côte d'Ivoire & Mauritania & Togo \\
\hline Equatorial Guinea & Mauritius & Uganda \\
\hline Eritrea & Mozambique & Zambia \\
\hline Eswatini / (Swaziland) & Namibia & Zimbabwe \\
\hline
\end{tabular}

uary 2000 through to December 2020. The rationale behind this date range selection is that global research on multimorbidity only started increasing in the early 2000 s, with $80 \%$ of studies published after $2010.5,24$ We therefore anticipate that before 2000 there were very few originating from sub-Saharan Africa, especially considering that even after 2010 only approximately $5 \%$ of research in this field comes from LMICs. ${ }^{24}$ Furthermore, the availability of ART after 2000 changed the HIV situation in the region, making it a manageable chronic condition, ${ }^{25}$ and considering the scope of research around HIV comorbidities and NCDs we deem this date range most appropriate for the sub-Saharan Africa context. We will apply no language limits on our literature search; studies in other languages will be translated to English.

We will exclude studies conducted among people below the age of 18 years and those from countries outside the sub-Saharan Africa region. Articles not reporting original peer-reviewed research such as abstracts, conference presentations, opinion reports and reviews will also not be included.

\section{SEARCH STRATEGY}

To identify appropriate and relevant studies for the review, the following databases will be searched; MEDLINE, Embase, Cumulative Index of Nursing and Allied Health Literature (CINAHL), Global Health, PsycINFO, African Journals Online and African Index Medicus. We will also hand search reference lists of relevant studies to identify further literature of interest. Additionally, we will use ProQuest Dissertation \& Theses, Web of Science, Google Scholar and Google search engine to identify grey literature such as government and institutional reports, theses and dissertations, as well as to track citations. Key authors for studies that meet eligibility criteria will be contacted if there is some missing information or full texts of their studies cannot be accessed.

A comprehensive search strategy and terms was developed by the team in collaboration with the University of Edinburgh Medical Librarian. We will conduct a broad search, adopting some search terms from previous systematic reviews on the topic. $7,8,26$ Search terms will include "multimorbidity”, “comorbidity”, "prevalence”, “sub Saharan Africa” and their synonyms. Medical subject headings (MeSH) and key texts words will be developed and combined with Boolean operators "AND” and/ “OR" across and within categories. A full search strategy for MEDLINE (Ovid) database is provided in Table 2, and will be tested and adapted to other databases.

\section{DATA COLLECTION AND ANALYSIS}

\section{SELECTION PROCESS}

For the first stage of the review, titles and abstracts of potentially eligible studies identified through electronic database searches, will be extracted to Endnote Library (EndnoteX9). Duplicates will be removed (both via Endnote function, and manual checking). Two reviewers will then independently review the titles and abstracts of the remaining studies to identify studies that potentially meet the inclusion criteria. If any discrepancies arise the two reviewers will discuss them and reach an agreement, with a third reviewer providing input. Selection of full text studies against the eligibility criteria will be carried out independently by the two reviewers. The third reviewer will also randomly check $10 \%$ of selected studies to check for consistency. Reasons for excluding studies will be documented throughout the process. A PRISMA flow diagram (Figure 1) ${ }^{27}$ will be used to outline the literature search and selection process. 
Table 2. MEDLINE Search Strategy via Ovid

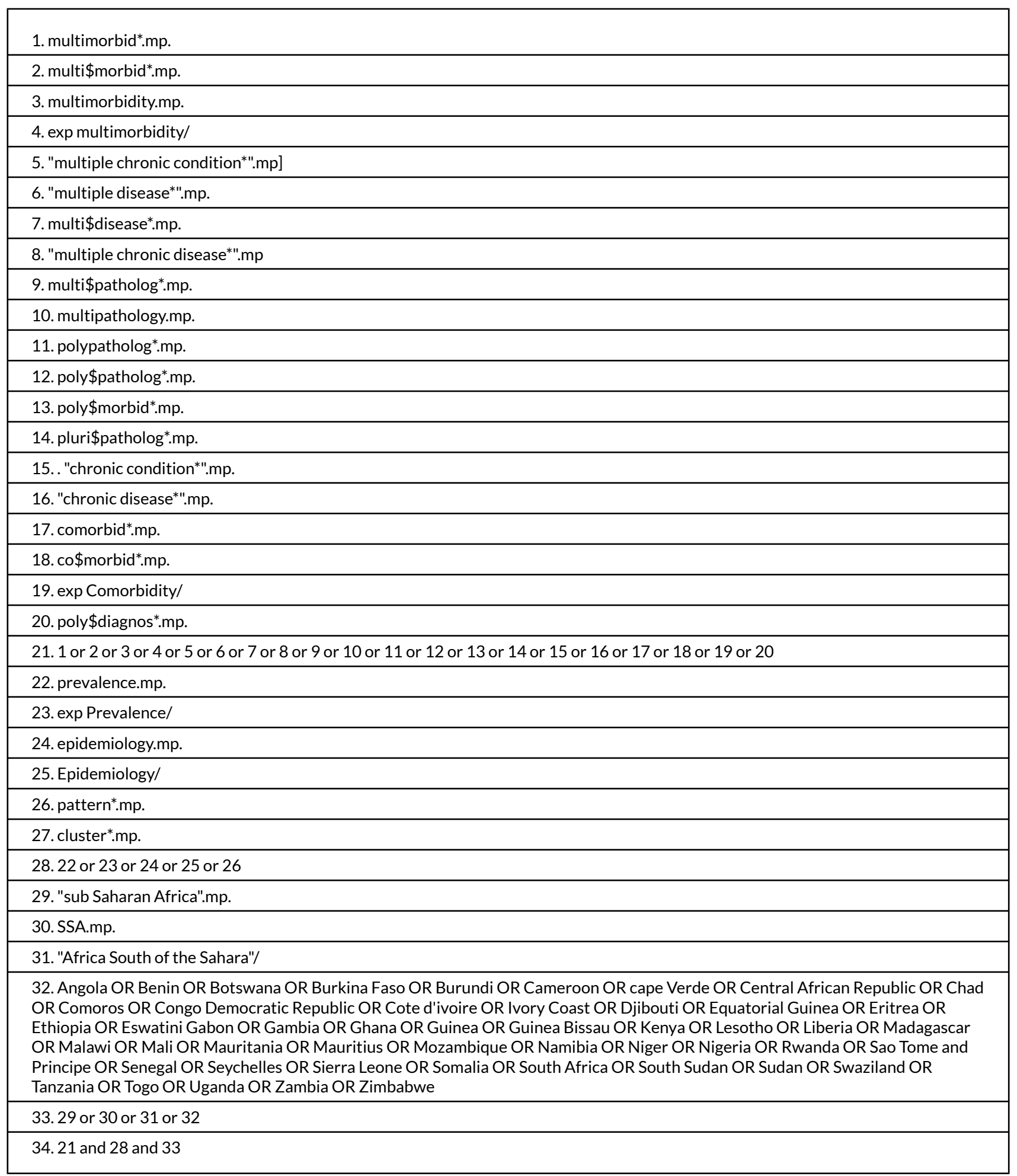

\section{DATA EXTRACTION}

Data extraction from selected studies will be carried out independently by two reviewers. In the event that there are disagreements, there will be discussions between the two reviewers and if a consensus is not reached the third reviewer will adjudicate. The team will develop a data extraction form which will be pre-tested. Key study characteristics are shown in Box 1.

\section{QUALITY ASSESSMENT}

We will evaluate the overall quality of individual studies using the Joanna Briggs Institute Prevalence Critical Appraisal Tool. ${ }^{28}$ This tool appraises external and internal validity of each individual study by addressing issues of representativeness of sample, recruitment of participants, identification of the condition, its measurement and statistical analysis among others. Two reviewers will independently assess the quality of included studies and disagreements will be 


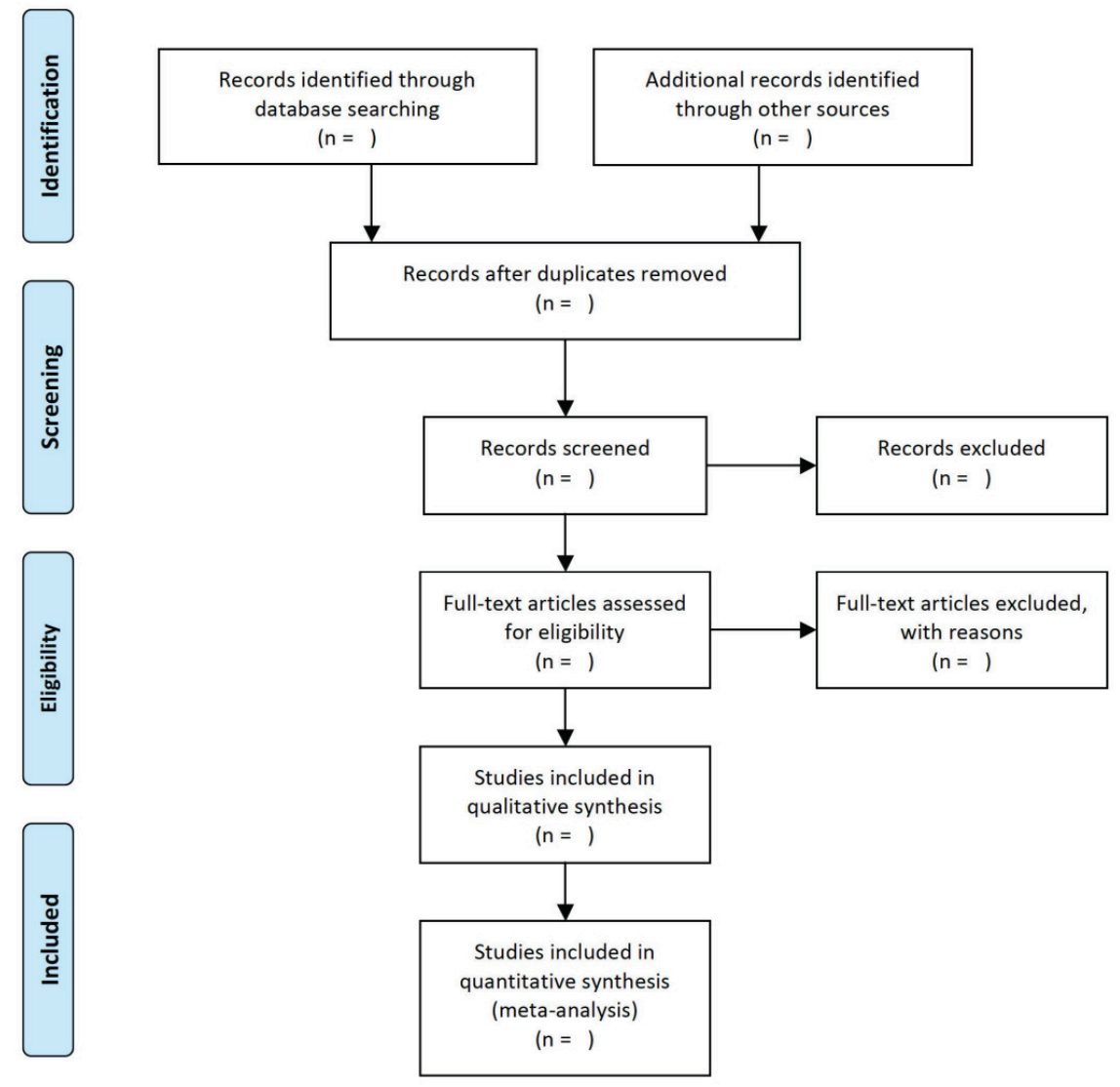

Figure 1. PRISMA flow diagram.

resolved through discussions with a third reviewer.

\section{Box 1. Study characteristics}

1. Authors, publication year and source of funding

2. Title and journal

3. Year of study

4. Country of study and location

5. Study design

6. Sample size

7. Study population characteristics (age, gender, any other measures such as rural/urban setting, education level)

8. Definition of multimorbidity

9. Measure of multimorbidity (simple diseases counts or weighted indices)

10. Source of multimorbidity data (self-reported, clinical records)

11. Number of conditions included in the study

12. Prevalence of multimorbidity

13. Disease combinations

\section{DATA ANALYSIS}

We will conduct a narrative synthesis of data from all included studies using "Guidance On The Conduct of Narrative Synthesis In Systematic Reviews". ${ }^{29}$ Studies will be clustered according to multimorbidity definition (2 or more chronic diseases, or 3 or more chronic diseases), setting (population based and facility based). We will generate evidence tables to summarise, descriptively, study and participants' characteristics. Characteristics of comorbidity studies versus multimorbidity will also be captured in table format. We anticipate that potential studies that will meet the inclusion criteria, are likely to be heterogeneous in different features such as study population, settings and data sources hence we expect that meta-analysis might not be suitable. ${ }^{30}$ However, if there are sufficient data that we are able to synthesise through meta-analysis, we will estimate a pooled prevalence of multimorbidity using random effects model together with their $95 \%$ confidence intervals (CI). Heterogeneity of studies will be assessed using the $\mathrm{I}^{2}$ statistic. An $\mathrm{I}^{2}$ value of $25 \%, 50 \%$ and $75 \%$ indicating low, medium and high heterogeneity, respectively. ${ }^{31}$ For any data with substantial heterogeneity, we will consider sub group analysis using mean age, sex, study setting, World Health Organisation Region in Africa, definition of multimorbidity. 


\section{ETHICAL CONSIDERATIONS}

This study does not require ethical approval as only secondary data will be assessed and does not involve interaction with the study subjects.

\section{CONCLUSION}

This review will identify and summarise evidence on the burden of multimorbidity in SSA taking into account the context of the region which is characterised by a double burden of communicable and non-communicable diseases. Results of this review will be published in peer reviewed journals and shared through relevant academic conferences.

\section{ACKNOWLEDGEMENTS}

We thank Marshall Dozier (Academic Support Librarian (Medicine), at the University of Edinburgh) for helpful discussion on search strategy design.

\section{FUNDING}

This is the first component of a $\mathrm{PhD}$ project which is examining the magnitude of multimorbidity and the common disease combinations in SSA.MRD is a PhD student at the Usher Institute, University of Edinburgh and is funded by the University of Botswana (UB). UB had no role in preparation, funding or decision to publish this protocol paper.

\section{AUTHORSHIP CONTRIBUTIONS}

MRD made substantial contribution to the conception of the protocol, drafting the manuscript and incorporated comments and suggestions from co-authors. DW and CC revised the drafts critically for important intellectual content and approved the final version for submission.

\section{COMPETING INTERESTS}

The authors completed the Unified Competing Interest form at www.icmje.org/coi_disclosure.pdf (available upon request from the corresponding author), and declare no conflicts of interest.

\section{CORRESPONDENCE TO:}

\author{
Mpho Refilwe Disang \\ Usher Institute \\ University of Edinburgh \\ Old Medical School \\ Teviot Place \\ Edinburgh, EH89AG \\ United Kingdom \\ M.R.Disang@sms.ed.ac.uk
}

Submitted: August 26, 2020 GMT, Accepted: October 10, 2020 GMT 


\section{REFERENCES}

1. Fortin M, Stewart M, Poitras ME, Almirall J, Maddocks $\mathrm{H}$. A systematic review of prevalence studies on multimorbidity: toward a more uniform methodology. Ann Fam Med. 2012;10(2):142-151. do i:10.1370/afm.1337

2. Xu X, Mishra GD, Jones M. Evidence on multimorbidity from definition to intervention: An overview of systematic reviews. Ageing Res Rev. 2017;37:53-68. doi:10.1016/j.arr.2017.05.003

3. Cassell A, Edwards D, Harshfield A, et al. The epidemiology of multimorbidity in primary care: a retrospective cohort study. British Journal of General Practice. 2018;68(669):e245. doi:10.3399/bjgp18X695 $\underline{465}$

4. Barnett K, Mercer SW, Norbury M, Watt G, Wyke S, Guthrie B. Epidemiology of multimorbidity and implications for health care, research, and medical education: a cross-sectional study. The Lancet. 2012;380(9836):37-43. doi:10.1016/S0140-6736(12)60 240-2

5. Catalá-López F, Alonso-Arroyo A, Page MJ, Hutton $\mathrm{B}$, Tabarés-Seisdedos R, Aleixandre-Benavent R. Mapping of global scientific research in comorbidity and multimorbidity: A cross-sectional analysis. PLoS One. 2018;13(1):e0189091. doi:10.1371/journal.pon e.0189091

6. Lefèvre T, d'Ivernois JF, De Andrade V, Crozet C, Lombrail P, Gagnayre R. What do we mean by multimorbidity? An analysis of the literature on multimorbidity measures, associated factors, and impact on health services organization. Rev Epidemiol Sante Publique. 2014;62(5):305-314. doi:10.1016/i.res pe.2014.09.002

7. Violan C, Foguet-Boreu Q, Flores-Mateo G, et al. Prevalence, determinants and patterns of multimorbidity in primary care: a systematic review of observational studies. PLoS One. 2014;9(7):e102149. doi:10.1371/journal.pone.010214 $\underline{9}$

8. Nguyen H, Manolova G, Daskalopoulou C, Vitoratou S, Prince M, Prina AM. Prevalence of multimorbidity in community settings: A systematic review and meta-analysis of observational studies. $J$ Comorb. 2019;9:2235042x19870934. doi:10.1177/2235 $\underline{042 \times 19870934}$

9. World Health Organization. Multimorbidity 2016. World Health Organization; 2016.
10. Diederichs C, Berger K, Bartels DB. The measurement of multiple chronic diseases--a systematic review on existing multimorbidity indices. J Gerontol A Biol Sci Med Sci. 2011;66(3):301-311. do i:10.1093/gerona/glq208

11. Murray CJL, Lopez AD, World Health Organization, World Bank, Harvard School of Public Health. The Global Burden of Disease: A Comprehensive Assessment of Mortality and Disability from Diseases, Injuries, and Risk Factors in 1990 and Projected to 2020. (Murray CJL, Lopez AD, eds.). World Health Organization; 1996. Accessed May 13, 2020. ht tps://apps.who.int/iris/handle/10665/41864

12. Global Burden of Disease. Global, regional, and national life expectancy, all-cause mortality, and cause-specific mortality for 249 causes of death, 1980-2015: a systematic analysis for the Global Burden of Disease Study 2015. Lancet. 2016;388(10053):1459-1544. doi:10.1016/s0140-673 $\underline{6(16) 31012-1}$

13. Gouda HN, Charlson F, Sorsdahl K, et al. Burden of non-communicable diseases in sub-Saharan Africa, 1990-2017: results from the Global Burden of Disease Study 2017. Lancet Glob Health.

2019;7(10):e1375-e1387. doi:10.1016/s2214-109x(1 9)30374-2

14. Mudie K, Jin MM, Tan, et al. Non-communicable diseases in sub-Saharan Africa: a scoping review of large cohort studies. J Glob Health. 2019;9(2):020409. doi:10.7189/jogh.09.020409

15. Patel P, Rose CE, Collins PY, et al.

Noncommunicable diseases among HIV-infected persons in low-income and middle-income countries: a systematic review and meta-analysis. AIDS (London, England). 2018;32(Suppl 1):S5-S20. doi:10.1097/OA D.0000000000001888

16. Dalal S, Beunza JJ, Volmink J, et al. Noncommunicable diseases in sub-Saharan Africa: what we know now. Int J Epidemiol. 2011;40(4):885-901. do $\underline{\mathrm{i}: 10.1093 / \mathrm{ije} / \mathrm{dyr} 050}$

17. El-Sadr WM, Goosby E. Building on the HIV platform: tackling the challenge of noncommunicable diseases among persons living with HIV. AIDS. 2018;32.

18. Chang AY, Gómez-Olivé FX, Payne C, et al. Chronic multimorbidity among older adults in rural South Africa. BMJ Global Health. 2019;4(4):e001386. d oi:10.1136/bmigh-2018-001386 
19. Moher D, Shamseer L, Clarke M, et al. Preferred reporting items for systematic review and metaanalysis protocols (PRISMA-P) 2015 statement. Systematic Reviews. 2015;4(1):1. doi:10.1186/2046-405 $\underline{3-4-1}$

20. Munn Z, Moola S, Lisy K, Riitano D, Tufanaru C. Methodological guidance for systematic reviews of observational epidemiological studies reporting prevalence and cumulative incidence data. Int J Evid Based Healthc. 2015;13(3):147-153. doi:10.1097/xeb.0 $\underline{000000000000054}$

21. Munn Z, Stern C, Aromataris E, Lockwood C, Jordan Z. What kind of systematic review should I conduct? A proposed typology and guidance for systematic reviewers in the medical and health sciences. BMC Medical Research Methodology. 2018;18(1):5. doi:10.1186/s12874-017-0468-4

22. Academy of Medical Sciences. Multimorbidity: $A$ Priority for Global Health Research.; 2018. Accessed May 21, 2020. https://acmedsci.ac.uk/policy/policy-pr ojects/multimorbidity

23. World Bank. World Bank Country and Lending Groups. World Bank; 2020. Accessed April 15, 2020. ht tps://datahelpdesk.worldbank.org/knowledgebase/arti cles/906519-world-bank-country-and-lending-groups

24. Xu X, Mishra GD, Jones M. Mapping the global research landscape and knowledge gaps on multimorbidity: a bibliometric study. Journal of global health. 2017;7(1):010414. doi:10.7189/jogh.07.010414

25. Coetzee L, Bogler L, De Neve JW, Bärnighausen T, Geldsetzer P, Vollmer S. HIV, antiretroviral therapy and non-communicable diseases in sub-Saharan Africa: empirical evidence from 44 countries over the period 2000 to 2016. J Int AIDS Soc.

2019;22(7):e25364. doi:10.1002/jia2.25364
26. Stirland LE, González-Saavedra L, Mullin DS, Ritchie CW, Muniz-Terrera G, Russ TC. Measuring multimorbidity beyond counting diseases: systematic review of community and population studies and guide to index choice. Bmj. 2020;368:m160. doi:10.11 36/bmj.m160

27. Moher D, Liberati A, Tetzlaff J, Altman DG, The PG. Preferred Reporting Items for Systematic Reviews and Meta-Analyses: The PRISMA Statement. PLOS Medicine. 2009;6(7):e1000097. doi:10.1371/journal.p med.1000097

28. Munn Z, Moola S, Riitano D, Lisy K. The development of a critical appraisal tool for use in systematic reviews addressing questions of prevalence. International journal of health policy and management. 2014;3(3):123-128. doi:10.15171/ijhp $\underline{\mathrm{m} .2014 .71}$

29. Popay J, Roberts H, Sowden A, et al. Guidance on the conduct of narrative synthesis in systematic reviews. A product from the ESRC methods programme Version. 2006;1:b92.

30. Pati S, Swain S, Hussain MA, et al. Prevalence and outcomes of multimorbidity in South Asia: a systematic review. BMJ Open. 2015;5(10):e007235. do i:10.1136/bmjopen-2014-007235

31. Higgins JP, Thompson SG. Quantifying heterogeneity in a meta-analysis. Stat Med. 2002;21(11):1539-1558. doi:10.1002/sim.1186 